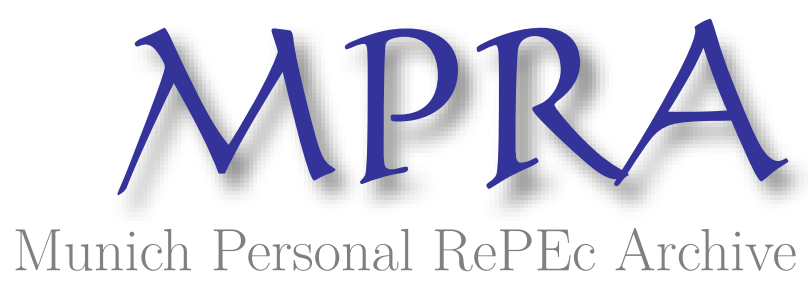

\title{
Delayed Default Dependency and Default Contagion
}

Balakrishna, B S

16 April 2007

Online at https://mpra.ub.uni-muenchen.de/14921/

MPRA Paper No. 14921, posted 30 Apr 2009 00:29 UTC 


\title{
Delayed Default Dependency and Default Contagion
}

\author{
B. S. BALAKRISHNA*
}

April 16, 2007; Revised: May 15, 2007

\begin{abstract}
Delayed, hence non-simultaneous, dependent defaults are discussed in a reduced form model. The model is a generalization of a multi-factor model based on simultaneous defaults to incorporate delayed defaults. It provides a natural smoothening of discontinuities in the joint probability densities in models with simultaneous defaults. It is a dynamic model that exhibits default contagion in a multi-factor setting. It admits an efficient Monte Carlo simulation algorithm that can handle heterogeneous collections of credit names. It can be calibrated to provide exact fits to CDX.NA.IG and iTraxx Europe CDOs just as its version with simultaneous defaults.
\end{abstract}

Credit derivatives sensitive to the default dependency of a portfolio of underlying assets are correlation products. The market standard for pricing such products is based on the Gaussian copula that has some well-known shortcomings. A consequence of this is the correlation smile implied from the market, an indication that the method is inadequate to price nonstandard products. Better models addressing these issues have been developed. Some recent work in this direction in a reduced form framework involves modeling the default intensities as in Joshi and Stacey [2005], Chapovsky, Rennie and Tavares [2006], Errais, Giesecke and Goldberg [2006], and modeling the loss distributions as in Bennani [2005], Sidenius, Piterbarg and Andersen [2005], Schönbucher [2005], Di Graziano and Rogers [2005], Brigo, Pallavicini and Torresetti [2006].

A semi-analytical parametric model for pricing such correlation products is presented in Balakrishna [2006]. It is based on simultaneous defaults and is in need of a generalization to allow for delayed defaults. This generalization takes us naturally to a reduced form model belonging to a class of jump-diffusion processes discussed in Duffie and Garleanu [2001]. Simultaneous default is a characteristic feature of the so called shock models based on Marshall-Olkin copula. They involve discontinuities in their joint probability densities. The present model can be viewed as an extension of such models offering a natural smoothening of those distributions. More importantly, it is a dynamic model that exhibits, in its multi-factor setting, clustering tendency of credit defaults known as default contagion. It admits an efficient Monte Carlo simulation algorithm applicable to homogeneous or heterogeneous collections. This can be used to provide exact fits to CDX.NA.IG and iTraxx Europe CDOs just as its version with simultaneous defaults.

First, in section 1, a multi-factor model based on instantaneous default dependency is reviewed. In section 2, it is generalized to a dynamic model with Poisson processes incorporating delayed defaults. In section 3, an efficient Monte Carlo algorithm applicable to homogeneous or heterogeneous collections is presented and used to calibrate the model to CDX.NA.IG and iTraxx Europe 5-year CDOs. In section 4, the ability of the model in a multi-factor setting to exhibit default contagion is investigated using its explicit solutions. Section 5 summarizes the results. Explicit solutions to the model are reviewed in Appendix A.

*Email: balak_bs@yahoo.co.in 


\section{Instantaneous Default Dependency}

Before looking at delayed defaults, it is worthwhile understanding the implications of simultaneous defaults. The model is based on event types termed factor names that are capable of causing joint defaults. It is assumed that there are $m$ of them all independent of each other. Concentrating on the arrivals of such independent events during an infinitesimal interval $(t, t+d t)$, we note that their joint probabilities are of order $(d t)^{2}$ or higher, and hence they could be treated individually.

Consider $n$ credit names, $i=1, \ldots, n$, with hazard rates $\lambda_{i}(t)$ 's. $\lambda_{i}(t) d t$ is the conditional probability that credit name $i$ defaults during $(t, t+d t)$ (rest of the names are not looked at). Let $\zeta_{r}(t) d t$ be the conditional probability of arrival of events of type $r$ during $(t, t+d t)$. Let $\gamma_{i r}(t)$ be the probability of finding credit name $i$ defaulted knowing that an event of type $r$ has arrived during $(t, t+d t)$. Picking up contributions from all factor names, along with a name-specific term $\bar{\lambda}_{i}(t)$, we can express $\lambda_{i}(t)$ as

$$
\lambda_{i}(t)=\bar{\lambda}_{i}(t)+\sum_{r=1}^{m} \gamma_{i r}(t) \zeta_{r}(t)
$$

Under the assumption that credit names are conditionally independent given a factor state, we can express the conditional probability density of joint defaults during $(t, t+d t)$ as

$$
p_{i j \ldots}(t)=\sum_{r=1}^{m}\left(\gamma_{i r}(t) \gamma_{j r}(t) \ldots\right) \zeta_{r}(t)
$$

There are no name-specific contributions here since they are of order $(d t)^{2}$ or higher. $p_{i j \ldots}(t) d t$ is the conditional probability that all the names in the list $\{i, j, \ldots\}$ default during $(t, t+d t)$ (unlisted names are not looked at). Related to this is $\pi_{i j \ldots}(t) d t$, conditional probability that at least one of the listed names defaults during $(t, t+d t)$ (unlisted names are not looked at), given by

$$
\pi_{i j \ldots}(t)=\sum_{k} \bar{\lambda}_{k}(t)+\sum_{r=1}^{m} \zeta_{r}(t)\left[1-\prod_{k}\left(1-\gamma_{k r}(t)\right)\right] .
$$

Here $k$ runs over only those names that are in the list $\{i, j, \ldots\}$. Note that the term under square brackets is the probability that at least one name in the list defaults during $(t, t+d t)$ given that an event of type $r$ has arrived during that interval. The instantaneous default correlation $\rho_{i j}(t)$ between credit names $i$ and $j$ is

$$
\rho_{i j}(t)=\frac{p_{i j} d t-\left(\lambda_{i} d t\right)\left(\lambda_{j} d t\right)}{\sqrt{\lambda_{i} d t\left(1-\lambda_{i} d t\right) \lambda_{j} d t\left(1-\lambda_{j} d t\right)}} \approx \frac{1}{\sqrt{\lambda_{i}(t) \lambda_{j}(t)}} \sum_{r=1}^{m} \gamma_{i r}(t) \gamma_{j r}(t) \zeta_{r}(t)
$$

Negative default correlations are not supported. If say $\lambda_{i}(t) \leq \lambda_{j}(t), \rho_{i j}(t)$ has an upper bound of $\sqrt{\lambda_{i}(t) / \lambda_{j}(t)}$ since $p_{i j}(t) / \lambda_{i}(t)$, probability of finding credit name $j$ defaulted during $(t, t+d t)$ knowing that $i$ has defaulted, should not exceed unity.

Under the assumption of instantaneous default dependency, the joint survival probability $Q$ up to times $t_{1}, \ldots, t_{n}$ ordered as $0=t_{0} \leq t_{1} \leq \ldots \leq t_{n}$ can be expressed as

$$
Q\left(t_{1}, \ldots, t_{n}\right)=\exp \left[-\sum_{i=1}^{n} \int_{t_{i-1}}^{t_{i}} d s \pi_{i \ldots n}(s)\right]
$$

where we have assumed a labeling of credit names according to their survival times for convenience. Note that $1-\pi_{i j \ldots}(t) d t$ is the conditional probability that none of the names in the list $\{i, j, \ldots\}$ 
default during $(t, t+d t)$ (unlisted names are not looked at). Hence, under our assumption of instantaneous default dependency, this expression for $Q$ can be obtained by building it up infinitesimally from $t=0$ to $t_{n}$ as a product of terms of the form $1-\pi_{i \ldots n}(t) d t$. It turns out that this joint survival probability belongs to a class of shock models based on Marshall-Olkin copula in a convenient representation and generalized to time dependent conditional probability densities.

Given the joint survival probability, we can get the joint survival and default probability $P_{i j \ldots}$. This is the probability that the names in the list $\{i, j, \ldots\}$ default before their times while the others survive up to their times. Probability that no names default before their times is of course given by $Q$ itself. To express others in terms of $Q$, let $Q_{i j \ldots}$ be constructed from $Q\left(t_{1}, \ldots, t_{n}\right)$ by setting $t_{i}, t_{j}, \ldots$ to zero for all the names in the list $\{i, j, \ldots\}$. Now, $P$ 's are obtained by alternately summing up various $Q$ 's, for instance, as

$$
P_{i j k}\left(t_{1}, \ldots, t_{n}\right)=Q_{i j k}-Q_{i j}-Q_{i k}-Q_{j k}+Q_{i}+Q_{j}+Q_{k}-Q .
$$

The dependence of $Q_{i j \ldots .}$ 's on the remaining times is not shown for simplicity. If those times are all the same, say $t$, we have from (5)

$$
Q_{i j \ldots}(t)=\exp \left[-\int_{0}^{t} d s \pi_{\neq i j \ldots}(s)\right]
$$

where $\{\neq i, j, \ldots\}$ lists out the names not in $\{i, j, \ldots\}$. For a homogeneous collection of credit names, $P_{\{\nu\}}$, the probability that $\nu$ names have defaulted before $t$ and the rest are not, simplifies to

$$
P_{\{\nu\}}=\left(\begin{array}{l}
n \\
\nu
\end{array}\right) \sum_{k=0}^{\nu}(-1)^{k}\left(\begin{array}{c}
\nu \\
k
\end{array}\right) Q_{[\nu-k]}=\left(\begin{array}{l}
n \\
\nu
\end{array}\right) \sum_{k=0}^{\nu}(-1)^{k}\left(\begin{array}{c}
\nu \\
k
\end{array}\right) \exp \left(-\int_{0}^{t} d s \pi_{[n-\nu+k]}(s)\right),
$$

where $Q$ and $\pi$ have only the number of names as subscripts. Using this result, the two-factor model can be calibrated to CDX.NA.IG and iTraxx Europe CDOs obtaining reasonable values for the parameters, as detailed in Balakrishna [2006].

Though default contagion is not apparent in this model because of simultaneity of defaults, clustering tendency can still be inferred. To see this consider $\chi_{\nu}^{(n)}$, the conditional probability of default during $(t, t+d t)$ of, say, credit name $n$, given the information that names $1, \ldots, \nu$ have defaulted during the same interval,

$$
\chi_{1}^{(n)}=\frac{\sum_{r=1}^{m} \gamma_{1 r} \gamma_{n r} \zeta_{r}}{\bar{\lambda}_{1}+\sum_{r=1}^{m} \gamma_{1 r} \zeta_{r}}, \quad \chi_{\nu>1}^{(n)}=\frac{\sum_{r=1}^{m}\left(\gamma_{1 r} \gamma_{2 r} \ldots \gamma_{\nu r}\right) \gamma_{n r} \zeta_{r}}{\sum_{r=1}^{m}\left(\gamma_{1 r} \gamma_{2 r} \ldots \gamma_{\nu r}\right) \zeta_{r}}
$$

This can be compared to $\chi_{\nu-1}^{(n)}$, conditional probability of default without the information about credit name $\nu$, for $\nu>1$. If $\chi_{\nu}^{(n)}>\chi_{\nu-1}^{(n)}$, it suggests that the likelihood of credit name $n$ being defaulted increases with the size of the cluster of names known to have defaulted. This is an indication that there is a tendency for defaults to cluster. As can easily be verified, this tendency is absent in a one factor model for $\nu>2$, but does exist in general for all defaults in a multi-factor model if the factor names can be ordered with $\gamma_{i r}$ 's, say, decreasing with increasing $r$ for all the credit names. This requirement on $\gamma_{i r}$ 's can be met if all the credit names in the collection couple to factor names in the same ordering of strength. We will defer an interpretation of these observations to later when we discuss jumps in hazard rates due to default contagion.

\section{Delayed Default Dependency}

The model of simultaneous defaults is based on the assumption of conditional independence of defaults at differing times. That is, if a credit name has survived an instant, an event at that 
instant does not have any further influence on the credit name. This ignores response times to events potentially causing defaults. Let us now allow for response times to such events and assume that they are exponentially distributed with means $\mu_{i r}^{-1}$ for each credit name $i$ and factor name $r$. This suggests that our expression for the hazard rates could perhaps be generalized to read like

$$
\lambda_{i}(t) \sim \bar{\lambda}_{i}(t)+\sum_{r=1}^{m} \int_{0}^{\infty} d s \mu_{i r} e^{-\mu_{i r} s} \gamma_{i r}(t-s) \zeta_{r}(t-s) .
$$

The effect of such a generalization would be to spread out simultaneous defaults to time periods of the order of the mean response times. The inverse mean response times, $\mu_{i}$ 's, are expected to be significantly larger than $\lambda_{i}$ 's.

The underlying dynamics of such a generalization is better explored by simulating factor names with Poisson processes $N_{r}(t)$ with intensity $\zeta_{r}(t)$ for $r=1, \ldots, m$. This involves replacing $\gamma_{i r} \zeta_{r} d t$ above by $-\operatorname{Ln}\left(1-\gamma_{i r}\right) d N_{r}$. This follows from the fact that $\zeta_{r} d t$ is of order $(d t)$ whereas $d N_{r}$ can take a value of one due to a jump in $N_{r}$ so that $\exp \left(\operatorname{Ln}\left(1-\gamma_{i r}\right) d N_{r}\right)$ has the expectation $\exp \left(-\gamma_{i r} \zeta_{r} d t\right)$. The resulting equation in differential form reads

$$
d \lambda_{i}(t)=\left[\phi_{i}(t)-\mu_{i} \lambda_{i}(t)\right] d t-\mu_{i} \sum_{r=1}^{m} \operatorname{Ln}\left(1-\gamma_{i r}(t)\right) d N_{r}(t),
$$

where $\phi_{i}(t)=d \bar{\lambda}_{i}(t) / d t+\mu_{i} \bar{\lambda}_{i}(t)$ and $\mu_{i r}$ 's are assumed to be independent of $r$. If $N_{r}(t)$ jumps up by one at time $t$ representing the arrival of an event of type $r, d N_{r}(t)$ causes $\lambda_{i}(t)$ to jump up by $-\mu_{i} \operatorname{Ln}\left(1-\gamma_{i r}(t)\right)$ at time $t$. The jump in $\lambda_{i}(t)$ decays exponentially until the arrival of another event at a later time (however, as we will see later, $\lambda_{i}(t)$ 's are no longer the true hazard rates).

The above process forms the basis of our analysis in what follows. If desired, the results of the analysis can easily be extended to handle time dependent $\mu_{i}$ 's. If the $r$ dependence of $\mu_{i r}$ 's is to be retained, one could consider differential equations of the above kind for each of the components $\lambda_{i r}(t)$ defined to add up to $\lambda_{i}(t)$ such that ${ }^{1}$

$$
\begin{aligned}
\lambda_{i}(t) & =\bar{\lambda}_{i}(t)+\sum_{r=1}^{m} \lambda_{i r}(t), \\
d \lambda_{i r}(t) & =-\mu_{i r} \lambda_{i r}(t) d t-\mu_{i r} \operatorname{Ln}\left(1-\gamma_{i r}(t)\right) d N_{r}(t) .
\end{aligned}
$$

It is also possible to work directly with the integral representation of (11) of the form (10) that can handle $\mu_{i r}$ 's dependent on factor indices.

Thus, from a model with simultaneous defaults belonging to a class of Marshall-Olkin copulas, introducing response times, we are naturally led to a reduced form model belonging to a class of jump-diffusion processes. These jump-diffusion processes are discussed in Duffie and Singleton [1999], except that our jump sizes are not exponentially distributed. Being related to the $\gamma$ parameters, it appears natural to keep the jump sizes fixed for the time being. As we will see later, this can still lead to distributed hazard rate jump sizes when there is insufficient information about the factor names causing the jumps. The model can be extended in various ways, for instance, to a fully specified jump-diffusion process or to a Cox process generalization of the Poisson processes. These will be studied elsewhere since our intention here is to explore the implications of event response times to default dependency that the model as such should be able to capture.

\footnotetext{
${ }^{1} \bar{\lambda}_{i}$ 's could be made stochastic as well, independent of $N_{r}$ 's, but our interest here is in the final joint probability distribution wherein that could just be a redefining of their time dependence. If desired, the number of factor names could be extended to include name-specific event types simulating $\bar{\lambda}_{i}$ 's.
} 
It is known that processes of the above kind have explicit solutions. For the joint survival probability up to time $t$ for credit names in the list $\{i, j, \ldots\}$ (the rest are not looked at), given by

$$
Q_{\neq i j \ldots}(t)=\mathrm{E}\left[\prod_{k} \exp \left(-\int_{0}^{t} d s \lambda_{k}(s)\right)\right]
$$

where the expectation is taken over the underlying Poisson processes, one obtains

$$
Q_{\neq i j \ldots}(t)=\exp \left(-\sum_{k} c_{k} \beta_{k}(t)-\int_{0}^{t} d s \pi_{i j \ldots}(s, t)\right) .
$$

Here and below $k$ runs over all the names in the list $\{i, j, \ldots\}$. The term involving $c_{k}=\lambda_{k}(0)-\bar{\lambda}_{k}(0)$ arises due to any contributions from events earlier to time zero and

$$
\begin{aligned}
\beta_{i}(s) & =\frac{1}{\mu_{i}}\left(1-e^{-\mu_{i} s}\right), \\
\pi_{i j \ldots}(s, t) & =\sum_{k} \bar{\lambda}_{k}(s)+\sum_{r=1}^{m} \zeta_{r}(s)\left[1-\prod_{k}\left(1-\gamma_{k r}(s)\right)^{\mu_{k} \beta_{k}(t-s)}\right] .
\end{aligned}
$$

If $\mu_{i r}$ is dependent on $r, \beta_{i}$ in these and the following expressions gets replaced by $\beta_{i r}$ defined for $\mu_{i r}$ and $c_{i}$ by $c_{i r}=\lambda_{i r}(0)$, with the term involving $c_{i r}$ getting summed over both $i$ and $r$.

The above $\pi$ density can be given an interpretation somewhat similar to that of our earlier section. In the limit $\mu_{k} \rightarrow \infty$ for all $k$, it does agree with our earlier result (3). For finite $\mu_{k}$ 's, it has a dependence on the "maturity" $t$ as well. This dependence drops out for constant $\gamma$ 's and $\zeta$ 's, as can be seen by rewriting the $\pi$ integral as

$$
\int_{0}^{t} d s \pi_{i j \ldots}(s, t)=\int_{0}^{t} d s\left\{\sum_{k} \bar{\lambda}_{k}(s)+\sum_{r=1}^{m} \zeta_{r}(t-s)\left[1-\prod_{k}\left(1-\gamma_{k r}(t-s)\right)^{\mu_{k} \beta_{k}(s)}\right]\right\} .
$$

Compared to its counterpart in the formalism of simultaneous defaults for constant $\gamma$ 's and $\zeta$ 's, the effect is just to use time varying $\gamma$ 's in (7) to compute the joint default probability distributions and various multi-name products. The effective $\gamma$ starts off at zero and increases to reach its constant value sufficiently after the mean response time.

One can also obtain an expression for the joint survival probability distribution given ordered times $0=t_{0} \leq t_{1} \leq \ldots \leq t_{n}$, that reads

$$
\begin{aligned}
Q\left(t_{1}, \ldots, t_{n}\right) & =\exp \left[-\sum_{i=1}^{n} c_{i} \beta_{i}\left(t_{i}\right)-\sum_{i=1}^{n} \int_{t_{i-1}}^{t_{i}} d s \pi_{i \ldots n}\left(s, t_{i}, \ldots, t_{n}\right)\right], \\
\text { where } \pi_{i \ldots n}\left(s, t_{i}, \ldots, t_{n}\right) & =\sum_{k=i}^{n} \bar{\lambda}_{k}(s)+\sum_{r=1}^{m} \zeta_{r}(s)\left[1-\prod_{k=i}^{n}\left(1-\gamma_{k r}(s)\right)^{\mu_{k} \beta_{k}\left(t_{k}-s\right)}\right] .
\end{aligned}
$$

This assumes a labeling of credit names according to their survival times for convenience. Note that the product terms in $\pi_{i \ldots n}$ and $\pi_{i+1 \ldots n}$ agree at $s=t_{i}$ moving over continuously from one integral to the other in the expression for $Q$. This smoothens out the discontinuities in the joint probability densities, a troubling feature of Marshall-Olkin like distributions in models involving simultaneous defaults.

An issue with the above jump-process is that the jumps are independent of $\lambda_{i}(t)$. One expects a credit name to pick up a jump depending on $\lambda_{i}(t)$, perhaps proportionately. This can be done, for instance, by appropriately attaching a factor $\lambda_{i}(t)$ to the jumps in the jump-process. The model then loses its solvability, but a Monte Carlo approach discussed next is better suited in any case. Generalizations of this kind deserve further study. 


\section{Monte Carlo Simulation}

For homogeneous collections, or for very small heterogeneous collections, joint probability distributions and prices of products dependent on them can be computed using equations (6), (14) and (16). This procedure however turns out to be both inconvenient and inflexible, and for heterogeneous collections that are not too small, highly inefficient. Fortunately, there exists an efficient Monte Carlo approach that makes use of the fact that factor names are represented in the model as Poisson processes. This simple simulation algorithm, better suited for both homogeneous and heterogeneous collections, reads as follows:

1. Draw $n+m$ independent uniform random numbers, $u_{i}, i=1, \ldots, n$ and $v_{r}, r=1, \ldots, m$. For each $r$ referring to a factor name, determine its first event arrival time given $v_{r}$. For each $i$ referring to a credit name, set the logarithm of survival probability $\operatorname{Ln} S_{i}(0)$ to zero and set $c_{i}(0)$ to any contribution from events earlier to time zero. Set $t_{o}=0$.

2. Given the $m$ event arrival times, determine the earliest arrival time $t$ and its event type $r$.

3. For each $i$ referring to a surviving credit name, update $\operatorname{Ln} S_{i}\left(t_{o}\right)$ to get $\operatorname{Ln} S_{i}(t)$ by subtracting

$$
F(t)=c_{i}\left(t_{o}\right) \beta_{i}\left(t-t_{o}\right)+\int_{t_{o}}^{t} d s \bar{\lambda}_{i}(s) .
$$

Check if $\operatorname{Ln} S_{i}(t)<\operatorname{Ln} u_{i}$ to determine whether this credit name defaults before time $t$. If so, determine its default time $t_{i}$ by solving $F\left(t_{i}\right)=\operatorname{Ln} S_{i}\left(t_{o}\right)-\operatorname{Ln} u_{i}$.

4. If $t$ is beyond the time horizon or there are no more surviving credit names, go to step 7 .

5 . For each $i$ referring to a surviving credit name, update $c_{i}\left(t_{o}\right)$ to get

$$
c_{i}(t)=c_{i}\left(t_{o}\right) e^{-\mu_{i}\left(t-t_{o}\right)}-\mu_{i} \operatorname{Ln}\left(1-\gamma_{i r}(t)\right) .
$$

6. Draw another independent uniform random number $v_{r}^{\prime}$. Given this, determine the time of next arrival of event type $r$ and update its event arrival time. Set $t_{o}=t$ and go to step 2 .

7. Given the default times, price the instrument. For the next scenario, go to step 1.

8. Average all the prices thus obtained to get a price for the instrument.

Event arrival times are obtained by equating their conditional probabilities of no arrival to $v_{r}$ 's. For instance, in step 6, given that an event of type $r$ has last arrived at time $t$, its next arrival time $t^{\prime}$ is obtained by solving $\exp \left(-\int_{t}^{t^{\prime}} d s \zeta_{r}(s)\right)=v_{r}^{\prime}$. In step 3 , if $t$ is beyond the time horizon, the time horizon can be used in place of $t$ to avoid solving for any default times beyond the horizon. If perturbing the parameters, say, during calibration or sensitivity analysis, one may have to be careful about discontinuities due to any default time crossing over maturity unless the number of scenarios is sufficiently large. The efficiency of the algorithm is dependent on the number of events arriving before maturity making it very efficient for low event arrival rates. Significant improvement in efficiency can be achieved by using quasi random sequences such as Sobol sequences to generate each of the independent uniform random numbers.

The algorithm can easily be adapted to handle time dependent or factor dependent $\mu$ 's. It has a limit for $\mu_{i} \rightarrow \infty$ providing an alternative to the semi-analytical approach to modeling simultaneous defaults. Expressing $c_{i}$ as $\mu_{i} c_{i}^{\prime}$ for each $i$ and taking the limit $\mu_{i} \rightarrow \infty$, one observes that $c_{i}^{\prime}(0)=0$ in step $1, c_{i}\left(t_{o}\right) \beta_{i}\left(t-t_{o}\right) \rightarrow c_{i}^{\prime}\left(t_{o}\right)$ in step 3 and $c_{i}^{\prime}(t)=-\operatorname{Ln}\left(1-\gamma_{i r}(t)\right)$ in step 5 . When solving for the default time in step 3 , one could first check if $\operatorname{Ln} S_{i}\left(t_{o}\right)-c_{i}^{\prime}\left(t_{o}\right)<\operatorname{Ln} u_{i}$ to determine whether default occurs exactly at the event arrival time $t_{o}$.

Consider a two-factor model with uniform $\gamma_{1}, \gamma_{2}$ parameters and a parameterization using $\zeta, \theta$ in place of $\zeta_{1}, \zeta_{2}$ such that

$$
\zeta_{1}=\frac{\gamma^{2}}{\gamma_{1}^{2}} \zeta \cos ^{2} \theta, \quad \zeta_{2}=\frac{\gamma^{2}}{\gamma_{2}^{2}} \zeta \sin ^{2} \theta, \quad \text { where } \frac{1}{\gamma}=\frac{1}{\gamma_{1}} \cos ^{2} \theta+\frac{1}{\gamma_{2}} \sin ^{2} \theta .
$$


Here $\gamma \zeta=\gamma_{1} \zeta_{1}+\gamma_{2} \zeta_{2}$, and $\gamma \sqrt{\zeta}$ is the magnitude and $\theta$ the angle of a two-dimensional vector with components $\gamma_{1} \sqrt{\zeta_{1}}$ and $\gamma_{2} \sqrt{\zeta_{2}}$. Table 1 shows the results of calibrating this model to CDX.NA.IG and iTraxx Europe 5-year CDOs on a homogeneous collection for different values of $\mu$. All model parameters are assumed to be time-independent except $\bar{\lambda}(t)$ that is assumed to follow $\bar{\lambda}(0) \exp \left(\kappa_{\lambda} t\right)$. The results are exact fits obtained with 25,000 scenarios using Sobol sequences and assuming no events before time zero. The procedure is very efficient taking just a couple of seconds for one simulation. Figure 1 displays the probability distributions computed with 25,000 and 100,000 scenarios. Even with as few as 25,000 scenarios, the procedure is able to capture the main features of the distribution. Figure 2 shows the effect of varying $\mu$ on the 5 -year joint probability distribution for fixed values of the other model parameters (the effect is significant just on the tail of the distribution since $\bar{\lambda}$ is not varied). It appears that $\mu$ has minimal effect on the distribution and the calibration results as long as it is not too small remaining of the order of 10 or more, or response times are not more than about a month or two.

An effective time dependence for $\bar{\lambda}(t)$ could be generated by modeling it as a diffusion process, for instance, along the lines of the Cox-Ingersoll-Ross model of interest rates. Extensions of this nature would also be useful in pricing products that are sensitive to dynamics of credit spreads. When $\bar{\lambda}(t)$ is calibrated to an exogenously supplied hazard rate curve, the model may not able to provide exact fits, but an acceptable best fit could still be possible.

\section{Default Contagion}

As noted earlier in the context of simultaneous defaults, multi-factor models of default dependency should be capable of exhibiting default contagion. However, models based on simultaneous defaults obscure such contagion features as jumps in hazard rates since all clustering of defaults takes place instantaneously. With delayed defaults, we should be able to see these features explicitly.

To start with, note that $\lambda$ 's are not true hazard rates. To see this, consider a credit name $i$ with survival probability $Q_{\neq i}(t)$ that can be deduced from (14). The true hazard rate as viewed at time zero is $h_{i}(t)=-d \operatorname{Ln} Q_{\neq i}(t) / d t$. For $\mu_{i} \rightarrow \infty$, it reduces to our earlier expression (1) as expected. For constant $\zeta_{r}$ and $\gamma_{i r}$, it simplifies to

$$
h_{i}(t)=c_{i} e^{-\mu_{i} t}+\bar{\lambda}_{i}(t)+\sum_{r=1}^{m} \zeta_{r}\left[1-\left(1-\gamma_{i r}\right)^{1-e^{-\mu_{i} t}}\right] .
$$

If there are contributions from events before time zero ensuring a sufficiently large $c_{i}$, this can be decreasing with respect to time. For smaller values of $c_{i}$, it can be increasing. Its time dependence is also controlled by that of $\bar{\lambda}_{i}(t)$.

To express the jumps in the hazard rates due to default contagion, say for credit name $n$, consider the probability density that credit names $1,2, \ldots, \nu$ have defaulted at their times $t_{1}, t_{2}, \ldots, t_{\nu}$ and the rest have survived up to their times, given by

$$
S_{\nu}\left(t_{1}, t_{2}, \ldots, t_{n}\right)=(-1)^{\nu} \frac{\partial^{\nu} Q\left(t_{1}, t_{2}, \ldots, t_{n}\right)}{\partial t_{1} \partial t_{2} \ldots \partial t_{\nu}} .
$$

Here times are ordered according to $t_{1}<t_{2}<\ldots<t_{n}$ and for convenience the credit names are labeled according to the same order. Using this, the hazard rate for credit name $n$, given that credit names up to $\nu-1$ have defaulted at their times and the rest have survived up to their times, can be written as

$$
h_{n}^{(\nu-1)}\left(t_{1}, t_{2}, \ldots, t_{n}\right)=-\frac{\partial}{\partial t_{n}} \operatorname{Ln} S_{\nu-1}\left(t_{1}, t_{2}, \ldots, t_{n}\right) .
$$


The jump in this hazard rate due to credit name $\nu$ defaulting is

$$
\Delta_{\nu} h_{n}=h_{n}^{(\nu)}-h_{n}^{(\nu-1)}=-\frac{\partial}{\partial t_{n}} \operatorname{Ln}\left[\frac{S_{\nu}}{S_{\nu-1}}\right] .
$$

If positive, this indicates that defaults are contagious, that for every credit name defaulting, the hazard rate of the name $n$ being observed jumps up. To know this jump at any time $t_{n}$, given all the survival and default information up to time $t \leq t_{n}$, all of $t_{\nu+1}, \ldots, t_{n-1}$ should be set to $t$. If interested in this jump just after the last credit name $\nu$ has defaulted, $t$ and $t_{n}$ should be set to $t_{\nu}$ as well. For the first credit name defaulting, we have

$$
\Delta_{1} h_{n}=-\frac{\partial}{\partial t_{n}} \operatorname{Ln}\left[-\frac{\partial}{\partial t_{1}} \operatorname{Ln} Q\right]
$$

Setting this to zero to understand the implications of conditional independence of defaults at differing times implies instantaneous default dependency as detailed in Balakrishna [2006].

Hazard rate jumps are, as one would expect, proportional to default correlation. This can be seen by rewriting the jumps as

$$
\Delta_{\nu} h_{n}=\frac{S_{\nu-1}}{S_{\nu}}\left[\frac{\partial_{n} \partial_{\nu} S_{\nu-1}}{S_{\nu-1}}-\frac{\left(-\partial_{\nu} S_{\nu-1}\right)}{S_{\nu-1}} \frac{\left(-\partial_{n} S_{\nu-1}\right)}{S_{\nu-1}}\right]=\sqrt{\frac{\partial_{n} S_{\nu-1}}{S_{\nu}}} \rho_{n \nu}^{(\nu-1)},
$$

where $\partial=\partial / \partial t$ and $\rho_{n \nu}^{(\nu-1)} \sqrt{d t_{n} d t_{\nu}}$ is the default correlation of credit name $n$ with $\nu$ for defaults during infinitesimal intervals $\left(t_{n}, t_{n}+d t_{n}\right)$ and $\left(t_{\nu}, t_{\nu}+d t_{\nu}\right)$ respectively, given the conditions implicit in $S_{\nu-1}$. For $\nu=1$, this relates to default correlation in the absence of default clustering and for $\nu>1$ to default correlation when default clustering is involved.

Explicit expressions for hazard rate jumps could be worked out, but it is convenient to make some assumptions to better elucidate the contagion effects. Some of these assumptions could be relaxed in a more elaborate analysis. To start with, let us assume that Poisson intensities are concentrated close to time zero, $\zeta_{r}(t)=\xi_{r} \delta\left(t_{+}\right)$, to understand its consequences to delayed defaults. It is then enough retain the following terms in $Q$ :

$$
Q \rightarrow \exp \left\{-\sum_{i=1}^{n} \bar{\lambda}_{i} t_{i}-\sum_{r=1}^{m} \xi_{r}\left[1-\prod_{i=1}^{n}\left(1-\gamma_{i r}\right)^{\mu_{i} \beta_{i}\left(t_{i}\right)}\right]\right\} .
$$

For simplicity, this assumes constant $\bar{\lambda}_{i}$ and $c_{i}=0$ for all $i$ ignoring events earlier to time zero. For the first default, that is, for credit name $\nu=1$ defaulting, we have

$$
\Delta_{1} h_{n}=e^{-\mu_{n} t_{n}} \frac{e^{-\mu_{1} t_{1}} \sum_{r=1}^{m} w_{r} v_{1 r} v_{n r}}{\bar{\lambda}_{1}+e^{-\mu_{1} t_{1}} \sum_{r=1}^{m} w_{r} v_{1 r}},
$$

where

$$
v_{i r}=-\mu_{i} \operatorname{Ln}\left(1-\gamma_{i r}\right), \quad w_{r}=\xi_{r} \prod_{i=1}^{n}\left(1-\gamma_{i r}\right)^{\mu_{i} \beta_{i}\left(t_{i}\right)} .
$$

To help identify the contagious effect on higher order jumps, let us further assume that the event probabilities under consideration are small. For the Poisson intensities concentrated close to time zero, this means that $\xi_{r}$ 's are small. $\bar{\lambda}_{i} / \mu$ 's that are expected to be small are assumed to be utmost of the order of $\xi_{r}$ 's. This lets us retain terms to their leading order only and use the approximation

$$
S_{\nu>1} \approx Q \prod_{i=1}^{\nu}\left[e^{-\mu_{i} t_{i}}\right] \sum_{r=1}^{m} w_{r}\left(v_{1 r} v_{2 r} \ldots v_{\nu r}\right) .
$$


In this approximation, the jump in the hazard rate due to the second default is

$$
\Delta_{2} h_{n}=e^{-\mu_{n} t_{n}}\left\{\frac{\sum_{r=1}^{m} w_{r} v_{1 r} v_{2 r} v_{n r}}{\sum_{r=1}^{m} w_{r} v_{1 r} v_{2 r}}-\frac{e^{-\mu_{1} t_{1}} \sum_{r=1}^{m} w_{r} v_{1 r} v_{n r}}{\bar{\lambda}_{1}+e^{-\mu_{1} t_{1}} \sum_{r=1}^{m} w_{r} v_{1 r}}\right\} .
$$

This can be shown to be positive for $\bar{\lambda}_{1} \geq 0$ if $v_{2 r}$ 's and $v_{n r}$ 's are, say, decreasing with increasing $r$. For higher order jumps, the result is

$$
\Delta_{\nu} h_{n}=e^{-\mu_{n} t_{n}}\left\{\frac{\sum_{r=1}^{m} w_{r}\left(v_{1 r} v_{2 r} \ldots v_{\nu r}\right) v_{n r}}{\sum_{r=1}^{m} w_{r}\left(v_{1 r} v_{2 r} \ldots v_{\nu r}\right)}-\frac{\sum_{r=1}^{m} w_{r}\left(v_{1 r} v_{2 r} \ldots v_{\nu-1 r}\right) v_{n r}}{\sum_{r=1}^{m} w_{r}\left(v_{1 r} v_{2 r} \ldots v_{\nu-1 r}\right)}\right\} .
$$

This can be shown to be positive as well if there are more than one factor names, and $v_{\nu r}$ 's and $v_{n r}$ 's are, say, decreasing with increasing $r$. These expressions involve terms looking similar to those involving $\gamma_{i r}$ 's discussed earlier in the context of simultaneous defaults and in terms of conditional probabilities. As expected, these jumps decay exponentially. It is this exponential decay that makes default clustering last not too long, unless of course there is another Poisson shock of the kind considered above at a later time. The distribution of defaults in a default cluster can be analysed, for instance for a homogeneous collection, using equations (8) and (16).

The requirement on $v_{\nu r}$ 's can be met if factor names can be ordered with $\gamma_{i r}$ 's decreasing with increasing $r$ for all the credit names. In this factor ordering, if $\gamma_{i r}$ 's, and hence $v_{i r}$ 's, are increasing with $r$ for some $r$ for some of the credit names, then it is possible that there are jumps in hazard rates that are negative. This suggests negative correlation between credit names having opposite order of factor couplings. Hazard rate jump of credit name $n$ due to credit name $\nu$ defaulting is, as already noted, proportional to its default correlation with credit name $\nu$ given the conditions implicit in $S_{\nu-1}$ that can be seen here for $\nu>2$ by writing

$$
\Delta_{\nu} h_{n} \propto \frac{\sum_{r=1}^{m} w_{\nu-1 r} v_{\nu r} v_{n r}}{\sum_{r=1}^{m} w_{\nu-1 r}}-\frac{\sum_{r=1}^{m} w_{\nu-1 r} v_{\nu r}}{\sum_{r=1}^{m} w_{\nu-1 r}} \frac{\sum_{r=1}^{m} w_{\nu-1 r} v_{n r}}{\sum_{r=1}^{m} w_{\nu-1 r}}
$$

where $w_{\nu-1 r}=w_{r}\left(v_{1 r} v_{2 r} \ldots v_{\nu-1 r}\right)$. Default correlation in the absence of default clustering is proportional to $\Delta_{1} h_{n}$ and is never negative. It is thus possible that a model with apparently only positive default correlations can exhibit negative correlations and hence negative hazard rate jumps when default clustering is involved. If this ever happens, it is likely at the initial stages of contagion, since, deep into the contagion, the jumps are governed by the larger $\gamma_{i r}$ 's that are expected to be associated with the dominant economic variables and factor ordered similarly for all the names.

In a one-factor model, hazard rate jumps are zero for $\nu>2$ to the leading order of our approximation. A possible interpretation is that, to this order, two defaults are enough to tell us about the cause of defaults, namely the only factor name governing default dependency. We would need two defaults, since the first default could have happened because of name-specific issues. Because $\bar{\lambda} \ll \mu$, it is unlikely that name-specific issues could have caused two defaults in a relatively short period of time of the order of $\mu^{-1}$. In a multi-factor model, hazard rate jumps are in general nonzero for all $\nu$, and in fact significant even for infinitesimal $\xi_{r}$ 's for $\nu \geq 2$. The interpretation is that, in our approximation, factor names can be held responsible for two or more defaults. But there is uncertainty about which factor name is responsible. With increasing number of defaults, terms with larger $v_{i r}$ 's within the summations in the above expressions gain increasing weight leading us to believe that factor names with higher couplings to credit names may be involved. Sufficiently high number of defaults is needed to convince us that it is due to the factor name whose coupling is the largest ${ }^{2}$. Any additional information about the cause of defaults exogenous to the model

\footnotetext{
${ }^{2}$ Default contagion as information driven has been discussed in Giesecke [2001] and Schönbucher [2003] in different contexts. For alternate mechanisms, see Davis and Violet [2000] and Jarrow and Yu [2000].
} 
is expected to just change the distribution of $\xi_{r}$ 's over $r$ without affecting the essence of these arguments. The model however needs to be enriched further by incorporating any feedback effect of defaults on the factor names to take into account any direct relationships between credit names.

\section{Conclusion}

In this article, the parametric model of default dependency based on simultaneous defaults discussed in Balakrishna [2006] is extended to accommodate response times to events potentially causing defaults. In the process, one is naturally led to a reduced form model belonging to a class of jump-diffusion processes that is free of simultaneous defaults. It can be viewed as an extension smoothening out the discontinuities in the joint probability densities in models based on instantaneous default dependency. It is a dynamic model and has the attractive feature that it is capable of exhibiting default contagion in a multi-factor setting that could explain clustering tendency of credit defaults. It admits an efficient Monte Carlo simulation algorithm applicable to homogeneous or heterogeneous collections of credit names, providing exact fits to CDX.NA.IG and iTraxx Europe CDOs just as its version with simultaneous defaults.

\section{A Explicit Solutions to the Model}

It is known that the following jump processes admit explicit solutions. Let us derive here some results that are relevant to the article. Consider $n$ jump processes for $\lambda_{i}, i=1, \ldots, n$ coupled together by common Poisson processes $N_{r}, r=1, \ldots, m$,

$$
d \lambda_{i}(t)=\left[\phi_{i}(t)-\mu_{i}(t) \lambda_{i}(t)\right] d t+\sum_{r=1}^{m} v_{i r}(t) d N_{r}(t)
$$

where $\phi_{i}(t)$ and $v_{i r}(t)$ are related to the model parameters as

$$
\phi_{i}(t)=\frac{d \bar{\lambda}_{i}(t)}{d t}+\mu_{i}(t) \bar{\lambda}_{i}(t), \quad v_{i r}(t)=-\mu_{i}(t) \operatorname{Ln}\left(1-\gamma_{i r}(t)\right) .
$$

Let us now look for an explicit solution to the following expectation taken over the common Poisson processes, with ordered times $t=t_{0} \leq t_{1} \leq t_{2} \leq \ldots \leq t_{n}$ :

$$
f\left(t, \lambda_{1}(t), \ldots\right)=\mathrm{E}_{t}\left[\prod_{i=1}^{n} \exp \left(-\int_{t}^{t_{i}} d s \lambda_{i}(s)\right)\right] .
$$

Its differential can be written down using Ito's calculus leading to the differential equation

$$
\frac{\partial f}{\partial t}+\sum_{i=1}^{n}\left[\left(\phi_{i}-\mu_{i} \lambda_{i}\right) \frac{\partial f}{\partial \lambda_{i}}-\lambda_{i} f\right]+\sum_{r=1}^{m} \zeta_{r}\left[f\left(t, \lambda_{1}+v_{1 r}, \ldots\right)-f\left(t, \lambda_{1}, \ldots\right)\right]=0 .
$$

This can be solved with an ansatz of the form

$$
f\left(t, \lambda_{1}, \ldots\right)=\exp \left[-\alpha(t)-\sum_{i=1}^{n} \beta_{i}(t) \lambda_{i}\right] .
$$

Equating coefficients of $f$ independent of $\lambda_{i}$ 's and those linear in $\lambda_{i}$ 's separately gives

$$
\begin{aligned}
\frac{d \beta_{i}}{d t}-\mu_{i} \beta_{i}+1 & =0, \\
\frac{d \alpha}{d t}+\sum_{i=1}^{n} \phi_{i} \beta_{i}+\sum_{r=1}^{m} \zeta_{r}\left[1-\prod_{i=1}^{n} e^{-\beta_{i} v_{i r}}\right] & =0 .
\end{aligned}
$$


These equations can be solved requiring $\beta_{i}(t)=0$ for $t \geq t_{i}$ that ensures continuity as $t$ is allowed to vary crossing various $t_{i}$ 's. The solutions are

$$
\begin{aligned}
\beta_{i}(t) & =\int_{t}^{t_{i}} d \tau \exp \left(-\int_{t}^{\tau} d s \mu_{i}(s)\right) \\
\alpha(t) & =\sum_{i=1}^{n} \int_{t_{i-1}}^{t_{i}} d s\left\{\sum_{k=i}^{n} \phi_{k}(s) \beta_{k}(s)+\sum_{r=1}^{m} \zeta_{r}(s)\left[1-\prod_{k=i}^{n} e^{-\beta_{k}(s) v_{k r}(s)}\right]\right\} .
\end{aligned}
$$

The solution to the expectation is, using model parameters in place of $\phi_{i}$ 's and $v_{i r}$ 's,

$$
\begin{aligned}
f\left(t, \lambda_{1}(t), \ldots\right) & =\exp \left[-\sum_{i=1}^{n}\left(\lambda_{i}(t)-\bar{\lambda}_{i}(t)\right) \beta_{i}(t)-\sum_{i=1}^{n} \int_{t_{i-1}}^{t_{i}} d s \pi_{i \ldots n}\left(s, t_{i}, \ldots\right)\right], \\
\text { where } \pi_{i \ldots n}\left(s, t_{i}, \ldots\right) & =\sum_{k=i}^{n} \bar{\lambda}_{k}(s)+\sum_{r=1}^{m} \zeta_{r}(s)\left[1-\prod_{k=i}^{n}\left(1-\gamma_{k r}(s)\right)^{\mu_{k} \beta_{k}(s)}\right] .
\end{aligned}
$$

In the article, $t$ is set to zero, $\mu$ 's are assumed to be time-independent for simplicity and the resulting $\beta_{k}(s)$ is treated as a function of $t_{k}-s$.

\section{References}

[1] Balakrishna, B. S. (2006), "A Semi-Analytical Parametric Model for Dependent Defaults", Working paper, http://defaultrisk.com/pp_crdrv128.htm.

[2] Bennani, N. (2005), "The Forward Loss Model: A Dynamic Term Structure Approach for the Pricing of Portfolio Credit Derivatives", Working paper, http://defaultrisk.com/ pp_crdrv_95.htm.

[3] Brigo, D., A. Pallavicini and R. Torresetti (2006), "Calibration of CDO Tranches with the Dynamical Generalized-Poisson Loss Model", Working paper, http://www.defaultrisk.com/ pp_crdrv117.htm.

[4] Chapovsky, A., A. Rennie and P. A. C. Tavares (2006), "Stochastic Intensity Modeling for Structured Credit Exotics", Working paper, http://defaultrisk.com/pp_crdrv_136.htm.

[5] Davis, M. and L. Violet (2000), "Modeling Default Correlation in Bond Portfolios", Working paper, Imperial College, London, 2000.

[6] Di Graziano, G. and C. Rogers (2005), "A Dynamic Approach to the Modeling of Correlation Credit Derivatives Using Markov Chains", Working paper, http://www.defaultrisk.com/ pp_crdrv_88.htm.

[7] Duffie, D. and N. Garleanu (2001), "Risk and the Valuation of Collateralized Debt Obligations", Financial Analysts Journal, 57, pp. 41-59.

[8] Duffie, D. and K. Singleton (1999), "Modeling Term Structures of Defaultable Bonds", Review of Financial Studies, 12, pp. 687-720.

[9] Errais, E., K. Giesecke and L. Goldberg (2006), "Pricing Credit from the Top Down with Affine Point Processes", Working paper, http://www.defaultrisk.com/pp_cdo_16.htm. 
[10] Giesecke, K. (2001), "Correlated Default with Incomplete Information", Journal of Banking and Finance, Humboldt Universitat Berlin, October 2001.

[11] Jarrow, R. and F. Yu (2000), "Counterparty Risk and the Pricing of Defaultable Securities", Journal of Finance, 56, pp. 1765-1799.

[12] Joshi, M. and A. Stacey (2005), "Intensity Gamma: A New Approach to Pricing Credit Derivatives", Risk Magazine, July 2006.

[13] Schönbucher, P. (2003), "Information Driven Default Contagion", Working paper, D-MATH, ETH Zürich, December 2003.

[14] Schönbucher, P. (2005), "Portfolio Losses and the Term Structure of Loss Transition Rates: A New Methodology for the Pricing of Portfolio Credit Derivatives", Working paper, http://defaultrisk.com/pp_model_74.htm.

[15] Sidenius, J., V. Piterbarg and L. Andersen (2005), "A New Framework for Dynamic Credit Portfolio Loss Modeling”, Working paper, http://defaultrisk.com/pp_model_83.htm.

Table 1: Two-factor exact fits to the five tranches of CDX.NA.IG and iTraxx Europe 5-year CDOs for the market quotes on June 2, 2006 for various values $\mu$ with 25,000 Monte Carlo scenarios. There are 125 names with a uniform recovery rate of $40 \%$. Premiums are paid quarterly. Interest rate is assumed at a constant 5\% for CDX.NA.IG and 3.5\% for iTraxx Europe CDOs. Equity tranche is quoted as an upfront fee in percent (plus 500bp per year running) and the other tranches are quoted as spreads per year in bp.

\begin{tabular}{|r|cccccc|}
\hline \multicolumn{7}{|c|}{ CDX.NA.IG 5y Series 6 } \\
\hline Tranches : & $0-3 \%$ & $3-7 \%$ & $7-10 \%$ & $10-15 \%$ & $15-30 \%$ & $0-100 \%$ \\
Quotes : & 30 & 97 & 20 & 10 & 5 & 40.3 \\
\hline$\mu$ & $\gamma_{1}(\%)$ & $\gamma_{2}(\%)$ & $\zeta(\mathrm{bp})$ & $\theta^{\circ}$ & $\bar{\lambda}(0)(\mathrm{bp})$ & $\kappa_{\lambda}$ \\
\hline 0.5 & 46.48 & 9.62 & 65.10 & 31.37 & 0.016 & 2.136 \\
1 & 40.23 & 8.38 & 58.10 & 30.93 & 0.016 & 2.135 \\
5 & 36.46 & 7.87 & 51.51 & 31.23 & 0.017 & 2.124 \\
10 & 36.14 & 7.84 & 50.60 & 31.28 & 0.017 & 2.125 \\
50 & 35.85 & 7.82 & 50.04 & 31.45 & 0.018 & 2.116 \\
100 & 35.83 & 7.82 & 49.95 & 31.38 & 0.018 & 2.114 \\
\hline \hline \multicolumn{7}{|c|}{ iTraxx Europe $5 y$ Series 5} \\
Tranches : & $0-3 \%$ & $3-6 \%$ & $6-9 \%$ & $9-12 \%$ & $12-22 \%$ & $0-100 \%$ \\
Quotes : & 23 & 70 & 19 & 9 & 4 & 31.0 \\
\hline$\mu$ & $\gamma_{1}(\%)$ & $\gamma_{2}(\%)$ & $\zeta(\mathrm{bp})$ & $\theta^{\circ}$ & $\bar{\lambda}(0)(\mathrm{bp})$ & $\kappa_{\lambda}$ \\
\hline 0.5 & 33.01 & 8.92 & 76.99 & 38.78 & 22.14 & 0.253 \\
1 & 28.72 & 7.79 & 69.04 & 38.94 & 21.98 & 0.257 \\
5 & 26.74 & 7.25 & 59.88 & 38.96 & 21.87 & 0.260 \\
10 & 26.29 & 7.22 & 59.08 & 39.23 & 21.83 & 0.261 \\
50 & 26.25 & 7.19 & 58.26 & 39.33 & 21.81 & 0.262 \\
100 & 26.22 & 7.19 & 58.16 & 39.34 & 21.81 & 0.262 \\
\hline
\end{tabular}


Figure 1: Logarithmic plot of the 5-year joint default probability distributions comparing the Monte Carlo results with 25,000 and 100,000 scenarios for 125 credit names assuming $\mu=10$ and the model parameters calibrated to 5-year iTraxx Europe CDOs.

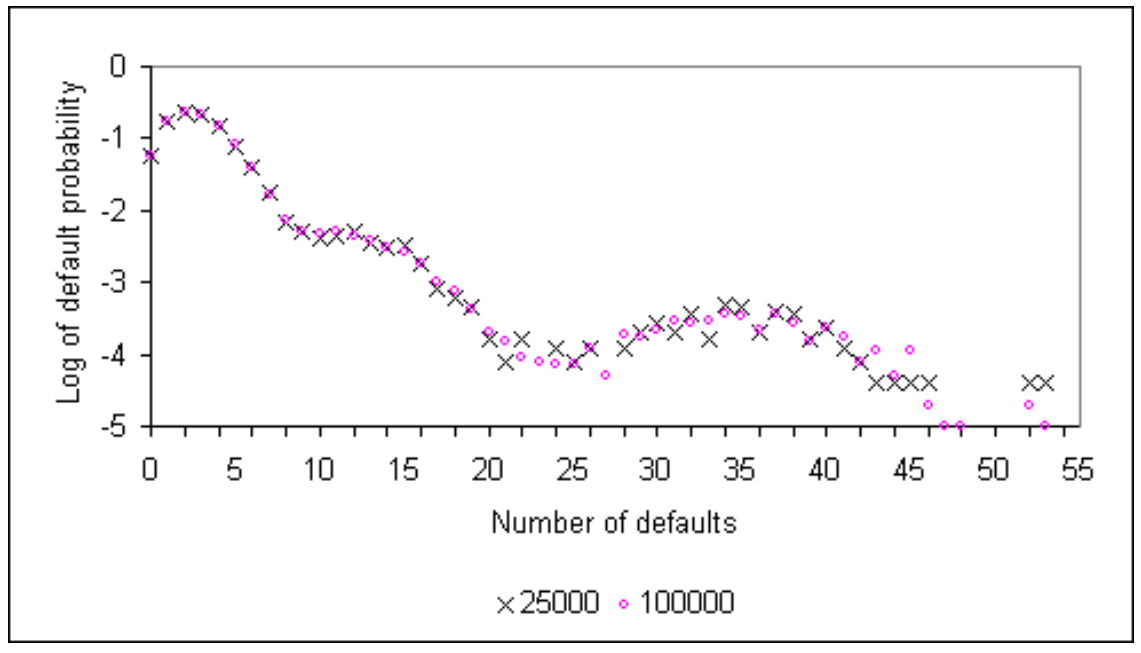

Figure 2: Logarithmic plot of the 5-year joint default probability distributions for various values of $\mu$ obtained with 1 million Monte Carlo scenarios for 125 credit names using fixed values for the other model parameters taken from the last row in Table 1.

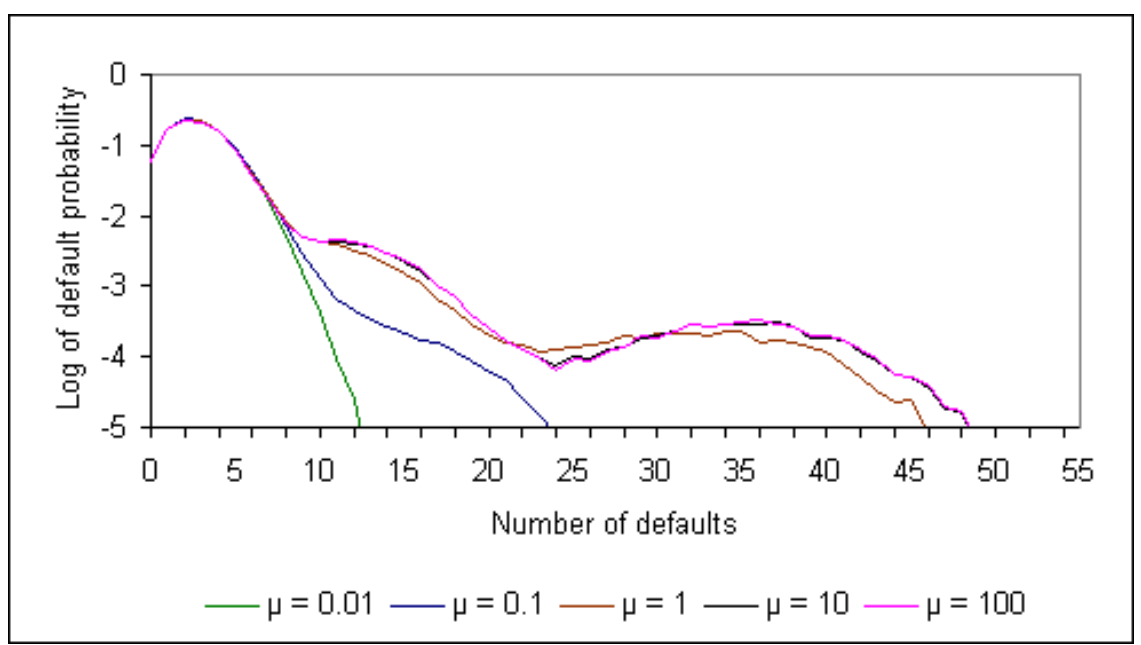

